\title{
Comparison of eight-week and twelve-week corticosteroid treatment regimens in children with idiopathic nephrotic syndrome; A clinical trial
}

\author{
Hossein Emad Momtaz ${ }^{1^{*}}$, Amin al Sadat Sharif $^{2}$, Ali Amri ${ }^{3^{\circledR}}$ \\ ${ }^{1}$ Division of Pediatric Nephrology, Besat Hospital, School of Medicine, Hamadan University of Medical Sciences, Hamadan, Iran \\ ${ }^{2}$ Division of Pediatric Nephrology, School of Medicine, Kashan University of Medical Sciences, Kashan, Iran \\ ${ }^{3}$ School of Medicine, Hamadan University of Medical Sciences, Hamadan, Iran
}

\section{A R T I C L E I N F 0}

Article Type:

Original

\section{Article History:}

Received: 3 January 2020

Accepted: 25 March 2020

Published online: 10 April 2020

Keywords:

Nephrotic syndrome

Children

Treatment

\begin{abstract}
A B S T R A C T
Introduction: Nephrotic syndrome (NS) is the commonest chronic glomerular disease in children. Idiopathic NS can perfectly be controlled using corticosteroids in most instances, but a significant relapse rate of NS is still a major problem. Several treatment protocols are suggested to decrease relapse rate of NS in children.

Objectives: The current clinical trial aimed at comparing the relapse rate between two 8 - and 12-week steroid treatment regimens.

Patients and Methods: In the current non-randomized, clinical trial, a total of 68 children with primary NS were enrolled. Oral prednisolone was administered to 34 patients for eight weeks $(2 \mathrm{mg} / \mathrm{kg} / \mathrm{d}$ and $1.5 \mathrm{mg} / \mathrm{kg} /$ alternate-day/each for four weeks) and other 34 patients for 12 weeks $(2 \mathrm{mg} / \mathrm{kg} / \mathrm{d}$ and $1.5 \mathrm{mg} / \mathrm{kg} / \mathrm{alternate-day/each} \mathrm{for} \mathrm{six} \mathrm{weeks).} \mathrm{A} \mathrm{one-year} \mathrm{follow-}$ up was completed for all the patients to evaluate relapse rate, steroid resistance, and steroid dependence.

Results: The remission rates were $47.1 \%$ and $73.5 \%$, respectively in children of the eight- and 12 -week treatment groups because the difference was significant $(\mathrm{P}=0.026)$. The frequent relapse rates in the eight- and 12 -week treatment groups were respectively $26.5 \%$ and $11.8 \%$. Steroid dependence rate was $17.6 \%$ and $8.8 \%$ in the eight- and 12 -week treatment groups respectively. The steroid resistance rates were respectively $8.8 \%$ and $5.9 \%$ in the eight- and 12-week treatment groups.

Conclusion: Twelve-week steroid treatment can significantly decrease the relapse rate in comparison with eight-week treatment because no significant difference in steroid resistance, steroid dependence, and frequent relapse between the two treatment protocols was observed. Trial registration: This study was approved by Iranian Registry of Clinical Trials (identifier: IRCT201404269014N33; https://www.irct.ir/trial/9472),
\end{abstract}

Implication for health policy/practice/research/medical education:

In a non-randomized, clinical trial to compare eight-week versus twelve-week corticosteroid treatment regimens in children with idiopathic nephrotic syndrome, we found twelve-week steroid treatment could significantly decrease the relapse rate in comparison with eight-week treatment because no significant difference in steroid resistance, steroid dependence, and frequent relapse between the two treatment protocols was observed.

Please cite this paper as: Emad Momtaz H, Al Sadat Sharif A, Amri A. Comparison of eight-week and twelve-week corticosteroid treatment regimens in children with idiopathic nephrotic syndrome; A clinical trial. J Renal Inj Prev. 2020; 9(3): e25. doi: 10.34172/jrip.2020.25.

\section{Introduction}

Nephrotic syndrome (NS) is a clinical condition caused by glomerular injury. It is characterized by edema, massive proteinuria, hypoalbuminemia, and hyperlipidemia. NS may be secondary to infectious diseases and systemic illnesses such as collagen vascular disease, malignancies, and primary renal disease (1).

In the pediatric age group, primary nephrotic syndrome 
(PNS) constitutes about $80 \%$ of cases $(1,2)$ with the annual incidence rate of $2-7$ per $10^{5}$ in children under 16 years old with higher prevalence in males (3). The most common type of PNS in children is minimal changes disease (MCD) that encompasses $90 \%$ of cases with favorable response to corticosteroid therapy (4). Children aged 1-12 years, without hypertension, gross hematuria, hypocomplementemia, permanent loss of renal function, and extra-renal manifestations are considered to have MCD while treatment could be started for them without renal biopsy. NS in children may be associated with complications such as infection, thromboembolism, and need for hospital admission to control edema and renal biopsy, which can cause inconvenience for patients and their families and impose a significant financial burden on the health system (5). Therefore, appropriate management of NS would be of great importance in clinical practice for pediatrics and pediatric nephrologists.

The first drug of choice to treat NS in children is steroids (6). About $90 \%$ of patients with MCD respond to steroids, while approximately $10 \%$ are resistant $(7,8)$. Approximately, a three-week steroid therapy is effective in $80 \%-90 \%$ of children, but most of the patients with NS have at least one recurrence.

The length of treatment with steroids has remained a matter of debate in children with NS. There is almost no standard treatment guideline all over the world due to lack of strong supporting evidence (9). To minimize the risk of relapse and cumulative steroid dose, different treatment protocols are employed. International study of kidney disease in children (ISKD) suggested a four-week regimen of prednisone $60 \mathrm{mg} / \mathrm{m}^{2} /$ day followed by another four-week regimen of $40 \mathrm{mg} / \mathrm{m}^{2}$ for three consecutive days per week (10). Another study suggested that daily administration of prednisone after the initial four weeks, if alternate-day prednisone given as a single dose, could decrease relapse rate in patients (11). Ehrisch et al concluded that six weeks daily treatment with $60 \mathrm{mg} / \mathrm{m}^{2}$ prednisone and six weeks alternate-day treatment with $40 \mathrm{mg} / \mathrm{m}^{2}$ prednisone would decrease relapse rate in comparison with four weeks daily and four weeks alternate-day treatment (12). Another study recommended a six-week regimen of $2 \mathrm{mg} / \mathrm{kg}$ prednisone daily followed by a further six-week regimen of $1.5 \mathrm{mg} / \mathrm{kg}$ alternate-day to treat children with NS (13).

The long-term side effects such as hypertension, hyperglycemia, osteoporosis, and growth failure should be considered when deciding on the duration of corticosteroid therapy (7).

\section{Objectives}

In this study, we tried to compare effect of two steroid treatment protocols (eight weeks versus twelve weeks) regarding the rate of relapse, steroid dependence and steroid resistance in children with NS.

\section{Patients and Methods}

\section{Study design}

A total of 68 children diagnosed with PNS referred to the pediatric nephrologist clinic enrolled in the study. Children with secondary NS, malnutrition, liver diseases, pancreatic insufficiency, celiac disease, Crohn's disease, and the individuals who previously treated with phenobarbital, rifampin, phenytoin, and isoniazid were excluded.

Patients in the present study were assigned to two groups: the first group underwent an eight-week prednisolone therapy and the second group was treated for 12 weeks with prednisolone, then at the end of the treatment, relapse, steroid dependence, steroid resistance, and frequent relapse were evaluated and compared between the two groups.

Assignment of the subjects to groups was performed based on the date of attendance at the clinic. In the beginning, the study objectives were explained to children's parents and written informed consent was taken. The first group was treated with prednisolone for a total duration of eight weeks (four weeks; $2 \mathrm{mg} / \mathrm{kg} / \mathrm{d}$ and then four weeks; $1.5 \mathrm{mg} / \mathrm{kg} /$ alternate-day) and the second group was treated with the same medication for a total duration of 12 weeks (six weeks; $2 \mathrm{mg} / \mathrm{kg} / \mathrm{d}$ and then six weeks; $1.5 \mathrm{mg} / \mathrm{kg} /$ alternate-day).

All the patients were monitored by testing their urine with dipsticks three times a week at home by parents and at least monthly by urine analysis to detect proteinuria. Patients were followed up for at least one year since resistance to prednisolone, frequent relapse, remission, and steroid dependence were documented in their records (Figure 1).

\section{Definitions}

Steroid resistance: Significant proteinuria continued despite a four-week administration of $2 \mathrm{mg} / \mathrm{kg} / \mathrm{d}$ prednisolone.

Steroid dependence: Relapse of proteinuria after reduction of dose or within two weeks after discontinuation of steroid therapy.

Frequent relapse: Two or more relapses in six months or four relapses in a year.

Relapse: Recurrence of proteinuria (dipstick testing $\geq 2+$ ) for three consecutive days.

Remission: Negative to trace urine protein for three consecutive days.

\section{Ethical issues}

This research was conducted following the Declaration of Helsinki principles. Informed written consent was obtained from the patients or their parents. This study was approved by the Ethics Committee of Human Research at Hamadan University of Medical Sciences (\#D/P/16/35/9/2932) and registered in Iranian Registry 


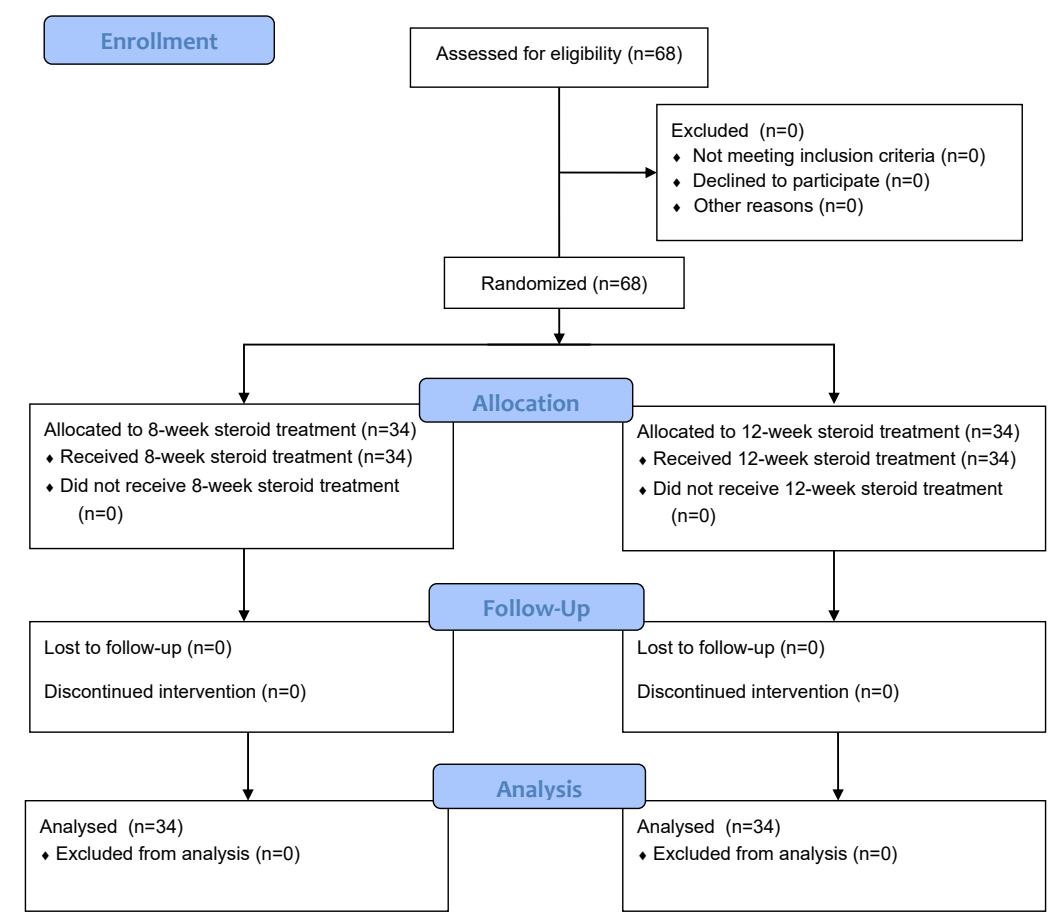

Figure 1. COSORT flow diagram of the study.

of Clinical Trials (identifier: IRCT201404269014N33; https:/www.irct.ir/trial/9472). Additionally, this study was extracted from the thesis of Aminosadat Sharif in Hamadan University of Medical Sciences.

\section{Data analysis}

Data were extracted from patients' records and transferred into forms. Then, the results of the two groups were statistically analyzed by SPSS software. Chi-square test was used for comparing gender, steroid resistance, steroid dependence, frequent relapses, remission rates and independent $t$ test for age distribution difference between two groups, while $P$ value $<0.05$ was considered as statistically meaningful.

\section{Results}

In this study, 68 children with PNS were recruited. In the eight-week treatment group, 22 of 34 patients $(64.7 \%)$ were male and $12(35.3 \%)$ female. On the other hand, in the 12 -week treatment group, 24 patients $(70.6 \%)$ were male and 10 (29.4\%) female, since the gender difference was not significant $(P=0.24$; Table 1$)$.

Table 1. Gender distribution in two groups

\begin{tabular}{lccc}
\hline \multirow{2}{*}{ Gender } & \multicolumn{2}{c}{ Duration of treatment } & \multirow{2}{*}{$\boldsymbol{P}$ value } \\
\cline { 2 - 3 } & 8-wk, No. (\%) & 12-wk, No. (\%) & \\
\hline Male & $22(64.7)$ & $24(70.6)$ & \\
Female & $12(35.3)$ & $10(29.4)$ & 0.24 \\
All & $34(100)$ & $34(100)$ & \\
\hline
\end{tabular}

The mean age of children in the eight- and 12-week treatment groups were $4 \pm 1.67$ and $4.9 \pm 1.97$ years respectively, while no significant difference between the groups regarding age distribution was observed $(P=0.83$; Table 2).

In the current study, $16(47.1 \%)$ and $25(73.5 \%)$ of the patients in the eight- and 12-week groups were in remission until the end of study, which showed a significant difference between the groups $(P=0.026$; Table 3$)$.

In the eight-week treatment group, six patients $(17.6 \%)$ and in the twelve-week group three $(8.8 \%)$ had frequent relapses. Difference was not statistically significant $(P=0.283$; Table 4).

In the eight-week treatment group, nine patients (26.5\%) and in the twelve-week group four patients

Table 2. Age distribution in two groups

\begin{tabular}{lccc}
\hline Group & No. & Mean age \pm SD $(\mathbf{y})$ & $\boldsymbol{P}$ value $^{\mathrm{a}}$ \\
\hline 8-wk treatment & 34 & $4 \pm 1.67$ & \\
12-wk treatment & 34 & $4.09 \pm 1.97$ & 0.83 \\
\hline a & & &
\end{tabular}

Table 3. Remission rate in two groups

\begin{tabular}{lccc}
\hline \multirow{2}{*}{ Group } & \multicolumn{2}{c}{ Remission, No. (\%) } & Chi-square test \\
\cline { 2 - 3 } & \multicolumn{1}{c}{ Yes } & No & \\
\hline $\begin{array}{l}\text { 8-wk treatment } \\
\begin{array}{l}\text { 12-wk } \\
\text { treatment }\end{array}\end{array}$ & $16(47.1)$ & $18(52.9)$ & $\begin{array}{c}\chi^{2}=4.97 \\
d f=1\end{array}$ \\
\hline
\end{tabular}


Table 4. Frequent relapse in the two groups

\begin{tabular}{lccc}
\hline \multirow{2}{*}{ Group } & \multicolumn{2}{c}{ Relapse, No. (\%) } & Chi-square test \\
\cline { 2 - 3 } & \multicolumn{1}{c}{ Yes } & No & \\
\hline 8-wk treatment & $6(17.6)$ & $28(82.4)$ & $\begin{array}{c}\chi^{2}=1.15 \\
d f=1\end{array}$ \\
12-wk treatment & $3(8.8)$ & $31(91.2)$ & \begin{tabular}{c}
$P$ value $=0.283$ \\
\hline
\end{tabular}
\end{tabular}

Table 5. Steroid dependence in the two groups

\begin{tabular}{lccc}
\hline \multirow{2}{*}{ Group } & \multicolumn{2}{c}{ Steroid dependence, No. (\%) } & \\
\cline { 2 - 3 } & Yes & No & \\
\hline 8-wk treatment & $9(26.5)$ & $25(73.5)$ & $\chi^{2}=2.37$ \\
$d f=1$ \\
12-wk treatment & $4(11.8)$ & $30(88.2)$ & $P$ value $=0.123$ \\
\hline
\end{tabular}

Table 6. Steroid resistance in two groups

\begin{tabular}{|c|c|c|c|}
\hline \multirow{2}{*}{ Group } & \multicolumn{2}{|c|}{ Steroid resistance, No. (\%) } & \multirow{2}{*}{ Chi-square test } \\
\hline & Yes & No & \\
\hline 8-wk treatment & $3(26.5)$ & 31 (73.5) & $\begin{array}{c}\chi^{2}=0.216 \\
d f=1\end{array}$ \\
\hline 12-wk treatment & $2(11.8)$ & $32(88.2)$ & $P$ value $=0.642$ \\
\hline
\end{tabular}

(11.8\%) became steroid dependent, however difference was not statistically significant $(P=0.123$; Table 5).

In the eight-week treatment group, three patients (26.5\%) and in the twelve-week group two patients (11.8\%) were steroid resistant. Difference was not statistically significant $(P=0.642$; Table 6$)$.

\section{Discussion}

One of the major therapeutic challenges in children with NS is the duration of corticosteroid therapy. Different treatment protocols are suggested to both reduce the risk of relapse and decrease steroid side effects, but there are controversies about optimum dose and duration of the treatment yet.

Webb et al conducted a study on 283 children presenting with the first episode of SSNS (steroid-sensitive nephrotic syndrome). The control group received prednisolone 60 $\mathrm{mg} / \mathrm{m}^{2} /$ day in weeks $1-4,40 \mathrm{mg} / \mathrm{m}^{2} /$ on alternate days in weeks 5-8 and placebo on alternate days in weeks 9-18 (total $2240 \mathrm{mg} / \mathrm{m}^{2}$ ). The intervention group received extended course prednisolone treatment: $60 \mathrm{mg} / \mathrm{m}^{2} /$ day in weeks $1-4$, then $60 \mathrm{mg} / \mathrm{m}^{2}$ on alternate days in weeks 5-18 tapering by $10 \mathrm{mg} / \mathrm{m}^{2}$ every 2 weeks (total $3150 \mathrm{mg} / \mathrm{m}^{2}$ ). Extended course (EC) prednisolone was administered to the intervention groups in weeks 1-4 followed by descending doses on alternate days in weeks 5-16, as well as every two weeks (total $3150 \mathrm{mg} / \mathrm{m}^{2}$ ). The time to first relapse, frequent relapse, steroid dependency, and need for other immunosuppressive therapies had no significant difference between the groups. They finally concluded that EC prednisolone therapy had no clinical benefits; however, the EC therapy was more cost-effective than the SC therapy (14).
Difference between the current study and that of Webb et al, was the number of patients (68 versus 283 ), duration of treatment (six and eight weeks versus eight and 16 weeks), employment of the placebo in their study, dosage of prednisolone, duration of follow-up (at least one year versus two years), and the final results.

Paul et al evaluated the difference between the eightweek standard corticosteroid regimen and the 12-week regimen in the treatment of NS. They divided 93 children with idiopathic NS into two groups; standard treatment $(n=46)$ and long-term treatment $(n=47)$. Patients in the standard group were treated with a four-week regimen of prednisolone followed by another four-week regimen with a lower dose on alternate days. The long-term group patients were treated with a six-week regimen of prednisolone followed by a lower dose on alternate days. Relapse in the next year was the primary outcome. In the current study, no significant difference was seen between the two groups in terms of relapse within the next year. Paul et al finally concluded that long-term prednisolone therapy does not influence the initial episode of steroidsensitive idiopathic nephrotic syndrome (15).

The difference between the current study and the study by Paul et al, was the number of patients (68 versus 93 ), the studied outcomes (prednisolone resistance, frequent relapse, relapse rate, remission, steroid dependence, and relapse within one year), and results (since 12week steroid treatment can significantly decrease the relapse rate compared to eight-week treatment, while long-term prednisolone therapy was not effective in the initial episode of steroid-sensitive idiopathic nephrotic syndrome). The dosage and type of medication, duration of follow-up, and number of samples in each group were the same in the two studies.

Sinha et al, in a study on 182 children with NS, investigated the differences in treatment outcomes between the three- and six-month corticosteroid regimens. Children were divided into two groups; after 12 weeks of standard therapy for both groups, one group received tapering prednisolone for another 12 weeks, while the other group received placebo for a further 12 weeks. The study results show that long-term prednisolone therapy in the initial phase did not affect the disease course in children with steroid-sensitive NS (16).

In a study by Mishra et al, 80 children were randomly treated with a five-month or a three-month prednisolone. After a one-year follow-up to record the frequency of relapses and side effects of steroid, the results showed a remarkable efficacy for the long-term prednisolone therapy in the initial episode of nephrotic syndrome, since the rate of relapse reduced, but the risk of steroid side effects did not increase (17).

Difference between our study and that of Mishra et al was the number of patients (68 versus 80 ), duration of treatment (12 and eight weeks versus three and five 
months), and outcomes, which were studied during follow-up; however, the final result was the same in the two studies.

Additionally, Beak et al reviewed the medical records of 99 patients; 54 patients received short-term treatment (an eight-week prednisolone therapy including a four-week daily administration plus a further four-week with a lower dose on every alternate day basis), while 45 patients received long-term steroid treatment (a 12-week prednisolone therapy including six weeks of daily administration plus a further six-week with a lower dose on every alternate day basis). They found that relapse rate was significantly lower one year after the first nephrotic syndrome episode in children receiving long-term treatment compared with the patients receiving short-term treatment (8). The type of medication, duration, dose of the drugs, results of the remission, and relapse frequency in the groups were the same in Beak et al and our study, while differences were the number of patients (99 versus 68 in our study), duration of follow-up (at least two years versus at least one year in our study). In addition, Beak et al had not assessed prednisolone resistance and dependency in their study.

\section{Conclusion}

According to the current study results, a higher remission rate and lower relapse rate was observed in children receiving a 12-week steroid therapy for the first episode of idiopathic nephrotic syndrome compared to the patients treated with an eight-week regimen of steroid, but no significant difference was observed between the treatment groups regarding steroid dependence, steroid resistance, and frequent relapse. However, further studies with larger sample sizes and longer follow-ups, as well as cost-efficacy and steroid side effects evaluations are needed.

\section{Limitations of the study}

Small sample size of two groups and short duration of follow up (one year) were limitations of our study. Larger studies with longer duration of follow up are recommended.

\section{Authors' contribution}

HEM participated in the study concept, design, critical revision and writing the final version of manuscript. AAS performed data collection. HEM and AA contributed to the initial draft. Data analysis was done by AA. All authors read and signed the final paper.

\section{Conflicts of interest}

The authors declare no conflict of interest.

\section{Ethical considerations}

Ethical issues including plagiarism and double publication have been completely observed by the authors. There was no study on animals in this research.

\section{Funding/Support}

This study was extracted from the thesis of Aminosadat Sharif in Hamadan University of medical sciences and was funded by deputy of research and technology of that University.

\section{References}

1. Chang JW, Tsai HL, Yang LY, Chen TJ. Epidemiology and predictors of end-stage renal disease in Taiwanese children with idiopathic nephrotic syndrome. J Epidemiol. 2012;22:517-22. doi: 10.2188/jea.JE20120033

2. Hinkes BG, Mucha B, Vlangos CN, Gbadegesin R, Liu J, Hasselbacher K, et al. Nephrotic syndrome in the first year of life: two-thirds of cases are caused by mutations in 4 genes (NPHS1, NPHS2, WT1, and LAMB2). Pediatrics. 2007;119:e907-e19. doi: 10.1542/peds.2006-2164.

3. Rüth EM, Kemper MJ, Leumann EP, Laube GF, Neuhaus TJ. Children with steroid-sensitive nephrotic syndrome come of age: long-term outcome. J Pediatr. 2005;147:202-7. doi: 10.1016/j.jpeds.2005.03.050.

4. Zhao J, Liu Z. Treatment of nephrotic syndrome: going beyond immunosuppressive therapy. Pediatr Nephrol. 2019 Mar 23. doi: 10.1007/s00467-019-04225-7.

5. Samuel SM, Dart A, Filler G, Bitzan M, Pinsk M, Mammen $\mathrm{C}$, et al. The Canadian childhood nephrotic syndrome (CHILDNEPH) study: report on mid-study feasibility, recruitment and main measures. BMC Nephrol. 2019;20:159. doi: 10.1186/s12882-019-1320-3.

6. Banerjee S, Basu S, Sen A, Sengupta J. The effect of vitamin D and calcium supplementation in pediatric steroid-sensitive nephrotic syndrome. Pediatr Nephrol. 2017;32:2063-2070. doi: 10.1007/s00467-017-3716-2.

7. Kliegman R, Stanton B, Geme JS, Schor NF, Behrman RE, Nelson WE. Nelson Textbook of pediatrics. Elsevier; 2020. p. 2758

8. Baek HS, Park KS, Kang HG, Ko CW, Cho MH. Initial steroid regimen in idiopathic nephrotic syndrome can be shortened based on duration to the first remission. Korean J Pediatr. 2015;58:206-10. doi: 10.3345/kjp.2015.58.6.206.

9. Noone DG, Iijima K, Parekh R. Idiopathic nephrotic syndrome in children. Lancet. 2018;392:61-74. doi: 10.1016/ S0140-6736(18)30536-1.

10. The primary nephrotic syndrome in children: identification of patients with minimal change nephrotic syndrome from initial response to prednisone-a report of the International Study of Kidney Disease in Children. J Pediatr. 1981;98:561564.doi:10.1016/s0022-3476(81)80760-3.

11. Ekka BK, Bagga A, Srivastava RN. Single- versus divideddose prednisolone therapy for relapses of nephrotic syndrome. Pediatr Nephrol. 1997;11:597-9. doi:10.1007/ s004670050344

12. Ehrich JH, Brodehl J. Long versus standard prednisone therapy for initial treatment of idiopathic nephrotic syndrome in children. Arbeitsgemeinschaft für Pädiatrische Nephrologie. Eur J Pediatr. 1993;152:357-61. doi: 10.1007/ bf01956754

13. Gipson DS, Massengill SF, Yao L, Nagaraj S, Smoyer WE, Mahan JD, et al. Management of childhood onset nephrotic syndrome. Pediatrics. 2009;124:747-57. doi: 10.1542/ 
peds.2008-1559.

14. Webb NJ, Woolley RL, Lambe T, Frew E, Brettell EA, Barsoum EN, et al. Sixteen-week versus standard eightweek prednisolone therapy for childhood nephrotic syndrome: the PREDNOS RCT. Health Technol Assess. 2019;23:1-108. doi: 10.3310/hta23260

15. Paul SK, Muinuddin G, Jahan S, Begum A, Rahman M, Hossain MM. Long versus standard initial prednisolone therapy in children with idiopathic nephrotic syndrome. Mymensingh Med J. 2014;23:261-7.
16. Sinha A, Saha A, Kumar M, Sharma S, Afzal K, Mehta $A$, et al. Extending initial prednisolone treatment in a randomized control trial from 3 to 6 months did not significantly influence the course of illness in children with steroid-sensitive nephrotic syndrome. Kidney Int. 2015;87:217-24. doi: 10.1038/ki.2014.240

17. Mishra OP, Thakur N, Mishra RN, Prasad R. Prolonged versus standard prednisolone therapy for the initial episode of idiopathic nephrotic syndrome. J Nephrol. 2012;25:394400. doi: 10.5301/jn.5000016.

Copyright ( $\odot 2020$ The Author(s); Published by Nickan Research Institute. This is an open-access article distributed under the terms of the Creative Commons Attribution License (http://creativecommons.org/licenses/by/4.0), which permits unrestricted use, distribution, and reproduction in any medium, provided the original work is properly cited. 